\title{
Cuba's Role in International Atomic Energy Agency Regional Cooperation in Cardiology
}

\author{
Amalia Peix MD PhD DSc, Berta García MS
}

\begin{abstract}
Cardiovascular diseases are the leading cause of death worldwide, and a health problem in low- and middle-income as well as high-income countries. They also constitute the main cause of death in Latin America, with ischemic heart disease as the principal cause in most countries of the region. In Cuba, heart disease is the first cause of death, followed by cancer and stroke. In its 2030 Agenda for Sustainable Development, the UN recognizes the importance of chronic non-communicable diseases, including cardiovascular diseases.
\end{abstract}

Cuba has participated actively as lead partner in design and implementation of the two regional technical cooperation projects conducted over the last six years by the International Atomic Energy Agency to address cardiovascular diseases in Latin American and Caribbean member states. These projects have generated greater interest among participating countries in the use of myocardial

\section{INTRODUCTION}

Cardiovascular disease (CVD) is the leading cause of death worldwide[1] and is a health problem not only for high-income countries. Due to the epidemiological transition; increased life expectancy, and increases in atherosclerotic risk factors such as diabetes mellitus, obesity, high blood pressure and smoking, CVD has also become a major health problem for low- and middleincome countries. These include those of Latin America and the Caribbean, a region with a 2016 population of over 640 million.[24] CVDs constitute the main cause of death in Latin America, with ischemic heart disease as the principal cause in most countries of the region.[3]

In Cuba, CVD is the first cause of death, followed by cancer and stroke; in 2017, it was responsible for 27,176 deaths (241.6 deaths per 100,000 population).[5] In men, the cancer mortality rate is slightly higher than that for CVDs (260.2 and 256.6 deaths per 100,000 population, respectively), while in women, it is the reverse, with respective rates of 188.1 and 226.8 deaths per 100,000 population.[5] The 2017 male:female ratio for cardiovascular mortality was 1.1.[5]

Primary prevention is essential for effective control of atherosclerotic risk factors and for reducing the burden of ischemic heart disease and other chronic diseases of atherosclerotic origin, such as

IMPORTANCE Cuba's contribution to the International Atomic Energy Agency's regional cardiology projects has fostered development of human resources and harmonized protocols both nationally and regionally, and demonstrated the importance of region-based cooperation for greater opportunities and more equitable access to resources also with important benefits to Cuba's own nuclear cardiology program. perfusion for dilated cardiomyopathy and coronary artery disease compared to other imaging techniques; disseminated knowledge about nuclear cardiology techniques and clinical applications in heart failure and coronary artery disease; and made important contributions to implementing harmonized, appropriate and safe clinical protocols. Cuba's contribution to the International Atomic Energy Agency's regional cardiology projects has fostered development of human resources and harmonized protocols both nationally and regionally, and demonstrated the importance of region-based scientific cooperation that ensures greater opportunities and more equitable access to resources. This participation has also accrued important benefits to Cuba's own nuclear cardiology program.

KEYWORDS Cardiovascular disease, myocardial perfusion imaging, nuclear cardiology, scientific cooperation, low-income populations, Latin America, Caribbean region, Cuba cerebrovascular disease and peripheral arterial disease. Cuba's Ministry of Public Health aims work in this direction, advised by national expert groups in the relevant specialties and supported by international organizations such as PAHO and WHO.

The UN has included chronic non-communicable diseases (NCDs), including CVDs, among targets in its 2030 Agenda for Sustainable Development. The UN Development Program has established 17 global Sustainable Development Goals (SDG).[6] SDG 3 ("Ensure healthy lives and promote well-being for all at all ages"), includes among its targets a $30 \%$ reduction of premature mortality from NCDs by 2030. To this end, WHO's Global Action Plan for Prevention and Control of Noncommunicable Diseases 2013-2020 includes a series of objectives aimed at NCD reduction, specifically at control of risk factors such as smoking, sedentary lifestyles and hypertension.[7]

At the same time, it is important for countries to have access to cutting-edge technologies for treating NCD patients in specialties such as cardiology, where nuclear medicine is fundamental. Application of nuclear medicine in Latin America and the Caribbean (LAC) varies markedly by country with regard to technological infrastructure, trained human resources, availability of radiopharmaceuticals and regulatory frameworks.

\section{CUBA'S ROLE IN INTERNATIONAL ATOMIC ENERGY AGENCY (IAEA) REGIONAL CARDIOLOGY PROJECTS}

As part of joint efforts by UN agencies, IAEA works systematically with member states to train medical personnel, physicists, radiochemists and technologists involved in nuclear medicine (considering training key to care quality); facilitates procurement of key medical equipment; and promotes communication among participants.

Cuba has been the lead partner in design and implementation of the two regional technical cooperation projects that IAEA has 
conducted in the last six years to address the problem of CVDs in Latin American and Caribbean member states. Cuba participated in the proposal of the main topics, project design, and organization and hosting of both first coordination meetings, and was the coordinator of the projects with the IAEA technical officer and project management officer. These projects were: Harmonization of Nuclear Cardiology Techniques in Patients with Congestive Heart Failure, with Emphasis on Chagas Cardiomyopathy (20122013) and Facing the High Incidence of Cardiovascular Diseases in Latin America and the Caribbean through Nuclear Cardiology (2016-2018)

Harmonization of Nuclear Cardiology Techniques in Patients with Congestive Heart Failure, with Emphasis on Chagas Cardiomyopathy Heart failure is a growing global epidemic affecting more than 15 million people worldwide-costly in terms of health, disability and mortality, as well as economically.[8,9] Coronary heart disease, diabetes mellitus and high blood pressure are the main risk factors. Dilated cardiomyopathy refers to a heterogeneous spectrum of myocardial diseases characterized by ventricular dilation and reduced myocardial contractility. Once patients become symptomatic, prognosis is relatively poor, with 25\% 1-year mortality and 50\% 5-year mortality.[10]

Among the causes of acquired dilated cardiomyopathy in Latin America, Chagas cardiomyopathy is one of the most frequent and is the leading cause of systolic heart failure in areas where Chagas disease is endemic.[11]

Given the morbidity and mortality from heart failure, as well as the considerable resources required to diagnosis and treat it, appropriate diagnosis and adequate prognostic evaluation are vital. The main objective of this regional project was to improve diagnosis in patients with heart failure, with special emphasis on Chagas cardiomyopathy, through the use of nuclear cardiology techniques, considering two fundamental aspects:

- Harmonization, which consists of the standardization of protocols for diagnosis, prognosis and risk assessment in patients with heart failure; and

- Training on and establishment of a methodology for evaluating intraventricular synchronism.

This was achieved through regional courses, visits from experts, training fellowships, a meeting of experts and standardization of nuclear cardiology protocols for managing patients with heart failure. An example of cooperation in the region was the active participation of local experts in regional courses, at no cost to the project. The first course on imaging, diagnosis and prognosis techniques in evaluation of heart failure took place in Cuba (Havana, July 2012), with participation by ten Cuban professors as well as international experts.

As a result of the project, 177 of the LAC region's professionals in the field of nuclear medicine and referring physicians in cardiology were trained through four regional courses and one conference on integrated cardiology imaging, the latter held in Vienna (September 2013).

Among nuclear techniques used in cardiology, gated myocardial perfusion scintigraphy with single photon emission computed tomography (gated SPECT) or with positron emission tomography is the only imaging technique capable of offering information about overall and regional ventricular function, presence of intraventricular synchronism and myocardial perfusion (including information on myocardial viability in the case of patients with previous myocardial infarction and poor ventricular function) in a single, reproducible test.[12-14]

Cuba participated in a meeting of 13 experts from the region in Rio de Janeiro, Brazil (September 2012), which resulted in publication of consensus-based guidelines on the important role for nuclear medicine in evaluating management of patients with heart failure.[12] Cuba also participated in the proposal preparation for a coordinated research project (Value of Intraventricular Dyssynchrony Assessment by Gated-SPECT Myocardial Perfusion Imaging in the Management of Heart Failure Patients Undergoing Cardiac Resynchronization Therapy),[15] developed with participation of 10 centers from 8 countries (2013-2018).

\section{Facing the High Incidence of Cardiovascular Diseases in Latin America and the Caribbean through Nuclear Cardiology Techniques The second research project was aimed at building capacity for early diagnosis and risk stratification of coronary artery disease (CAD), and providing guidance for evaluating patients before proceeding with interventional treatments.}

Visits and meetings of experts, regional courses, training grants and two publications all served to meet objectives regarding use of nuclear cardiology techniques in evaluating CAD patients. $[16,17]$ Several project country representatives participated in educational activities including: 7th Ibero-American Congress on Nuclear Cardiology (Havana, April 2016); International Conference on Integrated Medical Imaging in Cardiovascular Diseases (Vienna, October 2016); regional course on using nuclear cardiology techniques for diagnosis and risk stratification (Madrid, February 2017); regional quality assurance course and 26th Congress of the Latin American Biology and Nuclear Medicine Societies Santiago de Chile, November 2017); and a regional course on diagnostic imaging in cardiology and 8th Ibero-American Congress on Nuclear Cardiology (Bogotá, June 2018).

Country experts actively participated in the regional courses at no cost to the project and 42 specialists from the region (including 4 Cubans) attended the 2016 International Conference on Integrated Medical Imaging in Cardiovascular Diseases in Vienna. The American Society of Nuclear Cardiology collaborated with experts who participated in regional congresses and courses. In total, training was given to 487 nuclear medicine professionals and referring physicians in cardiology, 152 of them funded by IAEA. Ten percent of attendees participated in more than one event.

In Cuba, the first regional course was held in April 2016, in conjunction with the 7th Ibero-American Congress on Nuclear Cardiology and Cardiac Imaging, attended by 133 Cuban specialists as well as international participants. During 2017, for the purpose of disseminating the information and knowledge acquired by Cuban participants in the project's other regional courses, two workshops were held for national and international experts: Multimodal Imaging in Cardiology (160 participants, April 2017) and Imaging in Cardiology, from Diagnosis to Management (180 participants, December 2017). This knowledge also contributed to raising the level of the nuclear cardiology classes 
in the third iteration of the Nuclear Medicine Certificate Program taught in 2017 in Cuba.

Communication of the project's outcomes was another important aspect for both individual participating countries and the region as a whole. In Cuba, a video on CAD in women and the role of nuclear techniques in its diagnosis and management was created and disseminated through the IAEA website, major social networks and various specialized websites. Cuba also participated in an expert meeting (Costa Rica, September 2017) to prepare a consensus document, later published, on use of nuclear techniques in diagnosis, stratification and therapeutic practice.[18]

The regional projects were successful, generating awareness and interest among participating countries on the use of myocardial perfusion in evaluating patients with dilated cardiomyopathy and in CAD, compared to other imaging modalities and in particular in assessment of cardiac synchronism; disseminating knowledge about nuclear cardiology techniques and clinical applications in heart failure and in CAD; and contributing to advances in implementation of standardized, appropriate and safe clinical protocols.

A consensus document on the assessment of CAD patients with nuclear techniques, including the value of ischemia-guided therapeutic strategies was elaborated and published. All national health authorities of the participant countries were informed of the project's results as was the majority of their cardiology and nuclear medicine societies.

Due to the multidisciplinary nature of nuclear cardiology, various groups of professionals involved in its practice were included in the activities (not only nuclear medicine physicians and cardiologists, but also medical physicists, radiopharmacists and technologists). Training was expanded to include professionals from cities other than national capitals. One of the features with the greatest impact was the presence of experts sent by IAEA to national and regional conferences on both nuclear medicine and the medical specialties of referral practitioners.

The project revitalized the Ibero-American Conference on Nuclear Cardiology and Cardiac Imaging, an important regional framework for presentation of experiences in the region, held twice (Havana 2016 and Bogotá 2018).

What does participation in regional projects mean for Cuba? For our country, which has universal coverage and access to health services, participation in regional projects has provided its specialists and residents in cardiology, internal medicine and imaging, as well as medical physicists, radiopharmacists and technologists, with the opportunity to acquire knowledge that results in better care for heart patients.

The work of Cuban experts has contributed to better harmonization and increased use of nuclear techniques in cardiology, both in Cuba and regionally, as an example of collaboration among countries in Latin America and the Caribbean under the auspices of IAEA and national health authorities.

In low- and middle-income countries, careful selection of the appropriate diagnostic technique for each patient is especially important to achieve personalized, cost-effective medicine, as is evaluation of the impact of introducing new protocols or technologies. Almost two decades ago, the Economics of Noninvasive Diagnosis Study[19] of symptomatic women with suspected stable CAD showed that myocardial perfusion scintigraphy could be used as a tool to identify patients who should not have invasive coronary angiography, leading to cost reduction. Therefore, identifying a patient with CAD using nuclear techniques (or another imaging technique to detect ischemia, such as stress echocardiography or stress cardiac magnetic resonance, depending on availability and experience at a particular facility), enables an aggressive secondary prevention strategy to be initiated through optimal medical treatment according to clinical guidelines, as well as invasive treatment indicated by presence of ischemia in cases where necessary. Participation in these IAEA regional projects helps build capacities in this regard.

Issues persist requiring joint work by health authorities, scientific societies in the specialties involved and the Agency for Nuclear Energy and Advanced Technologies, with the advice of national groups. Among them:

- Implement an appropriate approach to use of nuclear techniques in multimodality imaging, with adequate costbenefit analysis. Training specialists in different imaging techniques will enable an interdisciplinary approach to patient care and better integration of appropriate indications for each technique.

- Continue implementation of quality control systems in nuclear medicine services to sustain improved care and increase patient satisfaction levels.

- Continue work to obtain approval of a medical specialty in nuclear medicine, although the country already has a nuclear medicine certificate program.

- Disseminate throughout Latin America and the Caribbean the importance of presenting and conducting projects related to regional health needs, specifically to reduce CVD morbidity and mortality. In this regard, cooperation among national health authorities, national and regional scientific societies and UN agencies such as IAEA, PAHO and WHO contribute to strengthening human and technological capacities in the region.

- More deeply study differences between men and women in relation to physiopathology of coronary heart disease and gender influence, a little-examined question nationally and regionally that leads to disparities in CAD treatment.[20,21] Along these lines, national and regional support is needed, including from UN agencies, with an approach that does not overlook the clinical and pathophysiological specificities of female patients.

\section{CONCLUSION}

Cuba's involvement in regional IAEA projects in cardiology has contributed to development of human resources and standardized protocols both nationally and in the Latin American and Caribbean region and demonstrated the importance of regional scientific cooperation that ensures greater opportunities and more equitable access to resources. $-1 /$ - 


\section{REFERENCES}

1. World Health Organization [Internet]. Geneva: World Health Organization; 2017 May 17. Newsroom. Cardiovascular diseases (CVDs); [updated 2015 Jan; cited 2018 Aug 23]. Available from: https://www.who.int/news-room/fact-sheets/de tail/cardiovascular-diseases-(cvds)

2. Statistics Division of the United Nations Economic Commission for Latin America and the Caribbean (UNECLAC).AnuarioEstadístico de América Latina y el Caribe 2016. Santiago de Chile: United Nations Economic Commission for Latin America and the Caribbean; 2017 Feb. 134 p. Spanish.

3. Lanas F, Serón P, Lanas A. Coronary heart disease and risk factors in Latin America. Glob Heart. 2013 Dec;8(4):341-8.

4. Pan American Health Organization; World Health Organization. Información y Análisis de Salud: Situación de Salud en las Américas: Indicadores Básicos 2015. Washington, DC.: Pan American Health Organization; 2015. Spanish.

5. National Health Statistics and Medical Records Division (CU). Anuario Estadístico de Salud 2017 [Internet]. Havana: Ministry of Public Health (CU); 2018 [cited 2018 Aug 23]. 207 p. Available from: http://bvscuba.sld.cu/anuario-estadistico-de -cuba/. Spanish.

6. United Nations. Sustainable Development Goals [Internet]. New York: United Nations; c2019 [cited 2018 Aug 23]. Available from: https://www.un.org/ sustainabledevelopment/sustainable-develop ment-goals/

7. World Health Organization [Internet]. Geneva: World Health Organization; c2019. Health Topics. Noncommunicable diseases and mental health. Events. Global action plan for the prevention and control of NCDs 2013-2020. Geneva: World Health Organization; [cited 2018 Aug 23]. Available from: http://www.who.int/nmh/events/ ncd_action_plan/en/

8. Cubillos-Garzón LA, Casas JP, Morillo CA, Bautista LE. Congestive heart failure in Latin America: the next epidemic. Am Heart J. 2004 Mar;147(3):412-7.

9. Najafi F, Jamrozik K, Dobson AJ. Understanding the 'epidemic of heart failure': a systematic review of trends in determinants of heart failure. Eur J Heart Fail. 2009 May;11(5):472-9.
10. Chen J, Normand SL, Wang Y, Krumholz HM. National and regional trends in heart failure hospitalization and mortality rates for Medicare beneficiaries, 1998-2008. JAMA. 2011 Oct 19;306(15):1669-78.

11. Bocchi EA. Heart failure in South America. CurrCardiol Rev. 2013 May;9(2):147-56.

12. Peix A, Mesquita CT, Páez D, Pereira CC, Félix R, Gutiérrez C, et al. Nuclear medicine in the management of patients with heart failure: guidance from an expert panel of the International Atomic Energy Agency (IAEA). Nucl Med Commun. 2014 Aug;35(8):818-23.

13. Dorbala S, Ananthasubramaniam K, Armstrong IS, Chareonthaitawee P, De Puey EG, Einstein $A J$, et al. Single photon emission computed tomography (SPECT) myocardial perfusion imaging guidelines: instrumentation, acquisition, processing, and interpretation. J Nucl Cardiol. 2018 Oct;25(5):1784-846.

14. Murthy VL, Bateman TM, Beanlands RS, Berman DS, Borges-Neto S, Chareonthaitawee P, et al. Clinical quantification of myocardial blood flow using PET: joint position paper of the SNMMI Cardiovascular Council and the ASNC. J Nucl Cardiol. 2018 Feb;25(1):269-97.

15. Peix A, Karthikeyan G, Massardo T, Kalaivani M, Patel C, Pabon LM, et al. Value of intraventriculardyssynchrony assessment by gated-SPECT myocardial perfusion imaging in the management of heart failure patients undergoing cardiac resynchronization therapy (VISION-CRT). J Nucl Cardiol. 2019 Jan 25. DOI: 10.1007/s12350-01801589-5. [Epub ahead of print].

16. Páez D, Peix A, Orellana P, Vitola J, Mut F, Gutiérrez $C$, et al. Current status of nuclear cardiology practice in Latin America and the Caribbean. J Nucl Cardiol. 2017 Feb;24(1):308-16.

17. Peix A, Páez D. Ischemic heart disease in Latin American women current perspective and call to action. J Nucl Cardiol. 2018 Oct 5. DOI: 10.1007/ s12350-018-1459-5. [Epub ahead of print]

18. Berrocal I, Peix A, Mut F, Shaw LJ, Karthikeyan G, Estrada Lobato E, et al. Appropriate use of noninvasive ischemia testing to guide revascularization decision making following acute ST elevation myocardial infarction in Latin American countries: results from an expert panel meeting of the International Atomic Energy Agency. Rev Esp Med Nucl Imagen Mol. 2018 Jul-Aug;37(4):23743. English, Spanish.

19. Shaw L, Hachamovitch R, Berman DS, Marwick TH, Lauer MS, Heller GV, et al. The economic consequences of available diagnostic and prognostic strategies for the evaluation of stable angina patients: an observational assessment of the value of precatheterization ischemia. J Am Coll Cardiol. 1999 Mar;33(3):661-9.

20. Virmani R, Burke AP, Farb A, Kolodgie FD. Pathology of the vulnerable plaque. J Am Coll Cardiol. 2006 Apr 18;47(8 Suppl):C13-8.

21. Baldassarre LA, Raman SV, Min JK, Mieres JH, Gulati M, Wenger NK, et al. Noninvasive imaging to evaluate women with stable ischemic heart disease. JACC Cardiovasc Imaging. 2016 Apr;9(4):421-35.

\section{THE AUTHORS}

Amalia Peix (Corresponding author: peix@ infomed.sld.cu), cardiologist with a doctorate in medical sciences and an advanced doctorate in sciences. Full professor, senior researcher, and coordinator, nuclear medicine working group, Cardiology and Cardiovascular Surgery Institute, Havana, Cuba.

Berta García, nuclear engineer with a master's degree in energy and nuclear technologies, and a diploma in foreign trade. National Assistant Liaison for technical cooperation with the International Atomic Energy Agency, Nuclear Energy and Advanced Technologies Agency, Ministry of Science, Technology and the Environment, Havana, Cuba

Submitted: January 9, 2019

Approved for publication: August 4, 2019 Disclosures: None 